\title{
Noticias viejas y nuevas sobre la inscripción de Obarra (CIL II 5840)
}

\author{
Old and new notices about the inscription of Obarra \\ (CIL II 5840)
}

\author{
Eduardo ORduña AZNAR \\ Javier VeLAZA FríAs*
}

\begin{abstract}
RESUMEN
ABSTRACT

En este trabajo revisamos la historiografía de la inscripción de Obarra (CIL II 5840).

El hallazgo de la única fotografía que se conoce de ella permite valorar nuevamente los antiguos testimonios y acercarnos a algunos detalles de su paleografía y de su datación.

PALABRAS CLAVE

Obarra, inscripción romana, CIL II 5840.

In this paper we revise the historiography of the inscription CIL II 5840 (Obarra). The finding of the only photography that we know of her allows to value again the former testimonies and to approach some details of his paleography and of his datation.

KEYWORDS

Obarra, Roman inscription, CIL II 5840.
\end{abstract}

Recibido el 17 de marzo de 2012. Aceptado el 18 de julio de 2012

La inscripción CIL II 5840 tiene una historia enrevesada. Vista por muy pocos estudiosos en el siglo XIX y perdida desde fecha imprecisa del siglo XX, arrastró desde siempre los problemas de una edición imperfecta y de unas noticias confusas sobre la localización. Cuando parecían haberse perdido las esperanzas de mejorar su estudio, ha venido a la luz una fotografía que permite asegurar su edición y precisar su paleografía y datación. En las siguientes páginas nos proponemos, pues, revisar su historiografía y, en la medida de lo posible, contribuir a su conocimiento epigráfico y textual ${ }^{1}$.

* Este trabajo se inscribe en los proyectos FFI2011-25113, FFI2009-13292-C03, FFI2011-25113 y en el Grup de Recerca Consolidat LITTERA (2009SGR105). Eduardo Orduña Aznar, Catedrático de Latín de Secundaria, Institut El Pont de Suert, c/Monestir de Lavaix, 13, 25520-El Pont de Suert (Lérida), eordunaaznar@gmail.com; Javier Velaza Frías, Catedrático de Filología Latina, Universidad de Barcelona, velaza@ub.edu.

1 No nos ocuparemos, sin embargo, de su interpretación como testimonio de la onomástica antigua, que ha dado lugar a múltiples referencias en la bibliografía sobre paleohispanística. 
La primera referencia a la pieza procede de un manuscrito de Abad y Lasierra², quien afirma haber visitado el lugar en 1772 buscando el Monasterio de Obarra — del que dice que «quizá se ignora todavía hasta el lugar de su situación»- y haber hallado «una Iglesia de Sta. María de Obarra, bastante capaz, y de buen arte, pero de poca antigüedad», que sorprendentemente no identifica con el monasterio. Tras describir un sepulcro que había en el interior, viene el texto que ha dado lugar, en nuestra opinión, a crear una cierta confusión sobre la procedencia de la lápida: «No encontrando la antigüedad que buscaba, claustro ni oficina alguna del Monasterio, me dediqué a examinar aquellas inmediaciones por no malograr el trabajo de haber penetrado semejantes asperezas: en lo alto de una colina, al poniente de dicha Iglesia, se hallan vestigios del antiguo Castro Ripacurcense, de que hablan sus escrituras; y en la Iglesia sobredicha hallé que tenían en el lugar de Ara una Lápida con una inscripción Romana, la cual me dijeron había estado sobre el portal del Castillo». La «Iglesia sobredicha» ha de ser sin duda el propio monasterio de Santa María de Obarra, ya que no menciona ninguna otra anteriormente. En cambio, es difícil saber a qué castillo se refiere. Abad y Lasierra presume que se trataría del Castrum Ripacurtiae conocido por las fuentes y cuya localización se desconoce, pero que probablemente estaría situado bastante más al oeste, hacia el Turbón ${ }^{3}$. Iglesias ${ }^{4}$ acepta la localización de Abad y Lasierra, precisando que las ruinas que visitó «cubrían la colina a Mediodía del mojón rocoso que trasdosa Obarra», esto es, la peña que domina el paso de La Croqueta. Sin embargo no hay nada en ese lugar que pueda relacionarse con alguna probabilidad con una construcción medieval, según hemos podido comprobar sobre el terreno.

La transcripción del epígrafe que ofrece Abad y Lasierra es la que sigue (Fig. 1):

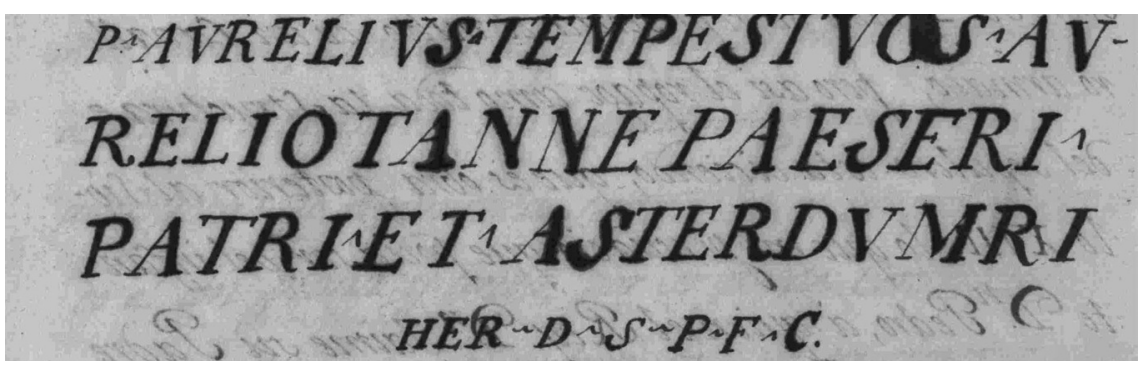

Fig. 1. Transcripción del epígrafe por ABAD Y LASIERRA, M.: op. cit., Biblioteca de la Real Academia de la Historia.

\footnotetext{
2 Biblioteca de la Real Academia de la Historia, Madrid, t. XXXVI, folio último, ABAD Y LASIERRA, Manuel, Manuscritos.

${ }^{3}$ Según un documento del s. XI citado por MARTíN DUQUE, Ángel, «El Monasterio de Obarra en los siglos XI a XIII», Príncipe de Viana, 227, (2002), p. 590, n. 54, el castillo en cuestión se encontraría en el límite occidental del ualle Ripacurcense: alodes in ualle Ripacurcense, de ipsum gradum de Aras usque in ipsum gradum de sancto Christoforo, et de Isauana usque in chastro de Ripacurza.

4 IGLESIAS COSTA, Manuel, Obarra. Jaca, Monografias del Instituto de Estudios Pirenaicos nำ105, 1975, p. 20)
} 
Conviene señalar que la lectura de Abad y Lasierra dista bastante de la realidad del texto: por un lado, lo distribuye en cuatro líneas cuando, como se verá, tiene seis. Además, en el final de lo que para él es la línea 4, comete un error de copia producido por una especie de «salto de igual a igual». Finalmente, no es riguroso al señalar las interpunciones. Sin embargo, como veremos más adelante, su lectura condicionará mucho la primera tradición del texto.

Habrá que esperar un siglo a que Fidel Fita ${ }^{5}$ se haga eco de la descripción de Abad y Lasierra, adornándola con nuevos datos y precisiones cuya fuente desconocemos: «Una roca, o peña muy ardua, que llaman La Croqueta, defiende allí naturalmente el paso; y estuvo coronada por el castro imponente, que contenía la basílica de San Pedro ${ }^{6}$, hoy desmantelado y totalmente en ruinas. De este castillo se extrajo y se bajó al templo de Obarra la inscripción marmórea». Y más adelante: «El Sr. Abad y La Sierra vio esta inscripción en el altar de San Pablo, que fue destruido con el desplome del campanario, algunos años há. El ilustrado párroco, D. José Bravo, me escribe que ha recogido el precioso epígrafe y lo ha pasado y guarda en la sacristía.» Lo cierto es que la única ruina documentada por esas fechas (1872) es la de los tramos de la bóveda más próximos a los pies de la iglesia, y por tanto en el extremo opuesto a los ábsides con sus altares, que seguían intactos cuando los fotografió Adolf Mas en 1907. A la derecha de la puerta principal se conserva la base de un campanario inconcluso ${ }^{7}$, de unos tres metros de altura. Sin embargo, Iglesias ${ }^{8}$ afirma que «se hundió la torre, y de las dos campanas que la poblaban, una se hizo pedazos al caer, y la otra fue instalada en el ventanal que había encima de la puerta de entrada" pero menciona a Antonio Zalacaín como párroco en 1872, en lugar del José Bravo mencionado por Fita en fechas muy poco posteriores. Por la posición de la base de campanario conservada, su hipotética caída hubiera tenido que afectar al tramo de nave situado a su lado, que sin embargo quedó intacto, como puede comprobarse por las fotografías de principios del siglo XX. La única posibilidad es, por tanto, que hubiera existido un campanario a los pies de la nave, como en Saint Aventin de l'Arboust, iglesia que Puig i Cadafalch ${ }^{9}$ considera estrechamente relacionada con la de Obarra, y que además hubiera habido en esa zona un altar, lo que resulta extraño. De Moner ${ }^{10}$ afirma que

5 FITA, Fidel, «Inscripciones romanas de la diócesis de Barbastro», Boletín de la Real Academia de la Historia, 4, (1884), pp. 211-227.

${ }^{6}$ Resulta sorprendente este detalle. ¿Podría referirse a la basílica de San Pedro del Vaticano, para dar idea de las dimensiones del castillo?

7 Según se afirma en PLADEVALL, Antoni (ed.), Catalunya Romànica, XVI. La Ribagorça. Barcelona, 1996, p. 348, aunque el principal argumento que se ofrece es la presencia de arcuaciones lombardas en ese tramo de muro. Sin embargo, esas arcuaciones se realizaron durante la restauración del templo, como demuestra una fotografía antigua de Ricardo del Arco. Naturalmente, la ausencia de arcos es lo normal, ya que se preveía que el campanario había de tapar ese muro, independientemente de que luego se terminara o no.

8 IGLESIAS COSTA, Manuel, El monasterio ribagorzano de Obarra. Barbastro, 1994, p. 40.

9 PUIG I CADAFALCH, Josep, L'arquitectura romànica a Catalunya II. Barcelona, 1909, p. 232.

10 DE MONER, Joaquín Manuel, «Nuestra Señora de Obarra en Ribagorza», Revista de Aragón, 29, (1879), p. 232. 
sus altares están dedicados a San Pedro, San Pablo, San Esteban y a la Santa Cruz, lo que al menos nos confirma un dato de Fita. Iglesias ${ }^{11}$ confirma que son esas las advocaciones del monasterio.

Fita, por lo tanto, no vio la pieza y, por lo que podemos inferir de la lectura que de ella ofrece, su informante el párroco Bravo tampoco le ofreció una transcripción. Eso explica que la lectura de Fita se base en la de Abad y Lasierra en lo tocante a la distribución de los párrafos y en las puntuaciones —sustituyendo los puntos abiertos de aquél por interpunciones triangulares dirigidas hacia arriba- Sólo en la palabra que Abad y Lasierra había leído como ASTERDVMRI Fita introdujo una conjetura en buena dosis correcta: su perspicacia de epigrafista le inclinó a pensar que en la forma final MARI debía de esconderse la palabra MATRI, en fórmula simétrica al patri anterior, y presupuso —según entendemos- que la palabra aparecería con nexos que Abad y Lasierra no habría llegado a comprender. Fita es también el primero que nos transmite unas medidas de la inscripción (46 x $60 \mathrm{~cm})$, que son las que consignará Hübner en el CIL, aunque probablemente no son exactas, como veremos (Fig. 2).

\section{P $\triangle$ AVRELIVS $\triangle$ TEMPESTIVOS $\triangle \mathrm{AV}$ RELIO $\triangle$ TANNEPAESERI $\triangle$ PATRI $\triangle$ ET $\triangle$ ASTERDV IARI $\triangle$ $\mathrm{HER} \triangle \mathrm{D} \triangle \mathrm{S} \triangle \mathrm{P} \triangle \mathrm{F} \triangle \mathrm{C}$}

Fig. 2. FITA, F.: op. cit. p. 221.

La traducción de Fita reza así: «Publio Aurelio Tempestivo, hijo heredero, erigió de su propio haber este monumento á su padre Aurelio Tannepéseris y á su madre Asterdu». El resto de su artículo se consagra a un análisis de la onomástica porque, como él mismo señalaba, «su estudio interesa en primer lugar a la ciencia de los lenguajes ibéricos».

Cuando Hübner recoge la inscripción en el Supplementum de CIL II tampoco ha tenido la oportunidad de verla. Sus datos dependen de Fita y así pues, de manera indirecta, de Abad y Lasierra: «In monasterio de Obarra, termino de Calvera, e castello vetusto la Croqueta dicto supra fluvium Isabena, in quo sunt relliquiae basilicae S. Petri; servatur iam in ecclesia monasterii. Est tabula marmorea alta $\mathrm{m}$. 0,46 , lata 0,60 , litteris optimis, punctis triangularibus». Su transcripción es idéntica a la de Fita, incluidos los nexos de la palabra MATRI ${ }^{12}$.

11 IGLESIAS COSTA, Manuel, Obarra..., p. 8.

12 De Hübner la tomó LOSTAL, Joaquín, Arqueología del Aragón romano. Zaragoza, 1980, p. 28. 
Poco después, Joaquín Costa ${ }^{13}$ se hace eco de los estudios anteriores de Abad y Lasierra, Fita y Hübner, y corrije correctamente la lectura, defectuosa desde el error inicial de Abad y Lasierra, a partir de un calco facilitado por un cirujano de Beranuy, por lo que parece segura su afirmación de que la inscripción se encontraba en ese momento custodiada en la sacristía, como afirmaba Fita (Fig. 3).

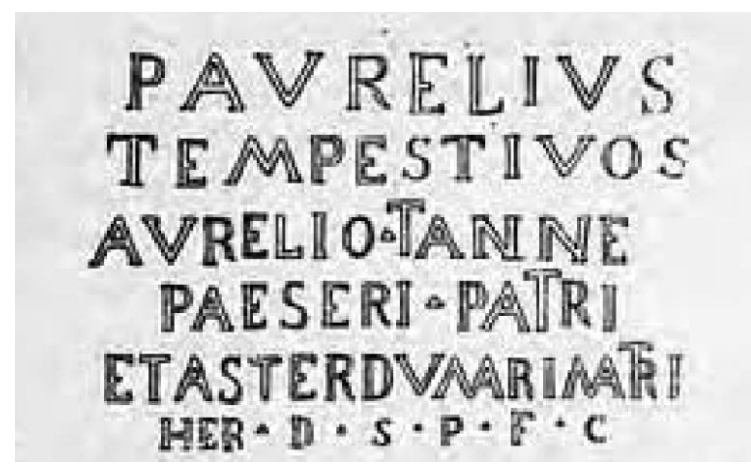

Fig. 3. COSTA, J.: op. cit. p. 90.

El dibujo de Costa es el primero en el que se reproduce correctamente la distribución de líneas; además, permite advertir algunos aspectos paleográficos del texto como los nexos de l. 5 o el módulo mayor de las letras T en las líneas 3 y 4. Sin embargo, no marca las interpunciones de las líneas 1 y 5 y parece mostrar una desigualdad en los módulos de algunas letras que no se corresponde con la realidad. Aunque no da las dimensiones, el calco que transmitió a Hübner permitió a éste rectificar las que había dado anteriormente: «lapis altus m. 0.42 , latus 0.56 , litteris saec. I exeuntis optimis, altis a m. 0.06 ad $0.025 » .^{14}$

No tenemos más datos de la pieza hasta la visita de la misión arqueológica del Institut d'Estudis Catalans en 1907; aunque se documenta la presencia de la inscripción, no la fotografían ni la dibujan, a diferencia de lo que hacen con la inscripción romana de Sopeira. La única mención aparece en una página del cuaderno de viaje de Mosén Gudiol, ${ }^{15}$ donde aparece un croquis del retablo del altar mayor, y a continuación se enumeran escuetamente otros objetos de interés: un par de sepulcros, una copia de la carta de fundación del monasterio, y la «Lápida de P. Aureli Tempestivos», sin indicar el lugar exacto, que hay que suponer que seguía siendo

${ }^{13}$ COSTA, Joaquín, «La inscripción hispano-latina de Obarra», Revista Critica de Historia y Literatura Españolas, 3, (1895), pp. 89-90.

${ }_{14}$ HÜBNER, Emil, «Additamenta nova ad corporis vol. II», Ephemeris Epigraphica, VIII, (1899), p. 430, núm. 175.

15 Reproducido en ALCOLEA, Santiago, La missió arqueològica de 1907 als Pirineus. Obra SocialFundació La Caixa, Barcelona, 2008, p. 111. 
la sacristía como sugiere el hecho de que se mencione a continuación de la carta fundacional. Ésta es la última mención a la lápida mientras estuvo en Obarra.

La siguiente referencia es de Del Arco, ${ }^{16}$ quien prácticamente se limita a transcribir lo ya dicho por Fita (op. cit.), salvo por la transcripción de la lápida, que es la correcta, y la alusión a su conservación en el Seminario de Barbastro, lo que nos da un margen 1907-1921 para el periodo en que la inscripción salió de Obarra definitivamente. Como ejemplo de la libertad con que se han ido transmitiendo los datos iniciales de Abad y Lasierra, mencionemos que, según Coromines, ${ }^{17}$ la inscripción se encontró al abrir el camino del paso de la Croqueta, y fue desenterrada al lado de un antiquísimo castrum.

La confusión de Abad y Lasierra sobre la propia identificación del monasterio románico ${ }^{18}$ permite arrojar ciertas dudas sobre sus informes acerca de la localización anterior de la inscripción. De su escrito no se deduce con total seguridad que él en persona viera los restos del castillo, y no podemos valorar la fiabilidad de la tradición oral que le fue transmitida, que en todo caso se refiere a un castillo sin duda próximo, pero sin precisar más. Sabemos que Obarra fue en el siglo X el núcleo del condado de Ribagorza, y que el monasterio fue elegido como panteón por varios de sus condes, por lo que algún castillo o residencia condal hubo de haber no lejos, pero desconocemos su ubicación.

En cuanto a la ubicación exacta de la inscripción en el monasterio antes de su traslado a la sacristía, hemos visto que Abad y Lasierra mencionaba efectivamente el uso como ara de la inscripción, aunque sin especificar de qué altar. Hay que descartar que se refiera a la próxima ermita de San Pablo, a unas decenas de metros al sur de Santa María, muy próxima por tanto, pero sin duda no lo bastante para verse afectada por una supuesta caida del campanario vecino, y cuya descripción separa claramente Abad y Lasierra en su relato. Si tomamos en consideración la alusión de Fita al altar de San Pablo, que pudo saber a través del párro$\mathrm{co}$, hay que señalar que una tabla del retablo del altar mayor, precisamente la única que se ha conservado (hoy en el Museo Diocesano de Barbastro) representa a San Pablo, y la del lado opuesto a San Pedro, y sabemos que ambos tenían altares en Obarra. En uno de los cuadernos de Puig i Cadafalch ${ }^{19}$ hay un croquis de la planta de uno de los ábsides laterales, aparentemente el situado al norte, y de su altar. Éste tenía una superficie de 83 × $92 \mathrm{~cm}$, sobre una base de 1,15 cm de alto y 80 x $63 \mathrm{~cm}$ de sección. Por lo tanto, si hemos de entender ara en el sentido que tiene en los altares cristianos, esto es, la piedra de consagración que cubre la cavidad donde se guarda la lipsanoteca o estuche que contiene reliquias y el

16 DEL ARCO, Ricardo, «Algunos datos sobre arqueología romana del Alto Aragón», Revista de Archivos, Bibliotecas y Museos, XLII, (1921), pp. 430-444.

17 COROMINES, Joan, «Una inscripció en basc ribagorçà del segle I, amb dos ideogrames». En Entre dos llenguatges, II. Barcelona, 1976, p. 132.

18 Es posible que lo que buscara Abad y Lasierra fuera la construcción visigótica anterior, de la que efectivamente no queda nada.

19 ALCOLEA, Santiago, op. cit., p. 57. 
acta de consagración, la inscripción romana sería demasiado grande para tal fin en un altar de ese tamaño, pero demasiado pequeña para ser ella misma la mesa del altar, pues sería más pequeña que la base. Por tanto, o bien el altar original era mayor, o bien la inscripción hubo de usarse como ara en otro altar, sea el altar mayor, u otro que pudo haber en la parte hundida de la nave, según la información transmitida a Fita por el párroco, aunque no es una ubicación normal para un altar en una iglesia como ésta.

En definitiva, lo único seguro es que la inscripción estuvo en Obarra utilizada como ara de altar al menos desde 1772, sin que sepamos con seguridad de qué altar se trata, después custodiada en la sacristía desde 1872, y desde Obarra fue trasladada a Barbastro en algún momento entre 1907 y 1921. En cuanto a la localización anterior, es dudosa la fiabilidad de los datos transmitidos por Abad y Lasierra, y el excelente estado de conservación de la placa más bien pone en duda que estuviera empotrada sobre el portal de un castillo.

Por lo que respecta a su localización actual, en una publicación reciente de fotografías de Ricardo del $\operatorname{Arco}^{20}$ se menciona el Museo Diocesano de Barbastro como lugar en que se tomó la fotografía única fotografía conocida de la inscripción ${ }^{21}$. Del $\mathrm{Arco}^{22}$ afirmaba que la inscripción se encontraba entonces en el Seminario de Barbastro, afirmación que recoge Pons, ${ }^{23}$ pero ni en aquel artículo ni después en 1942 en el Catálogo monumental de la provincia ${ }^{24}$ incluye foto alguna. A pesar de las pesquisas que hemos realizado, no consta que la inscripción figure entre los fondos del Museo Diocesano de Barbastro ni tampoco en el Seminario de la misma ciudad. Iglesias ${ }^{25}$ afirma que la inscripción «se conservó hasta época reciente y desapareció durante la guerra civil de 1936». Su testimonio tiene especial valor, pues Manuel Iglesias Costa, además de historiador y estudioso del arte románico, fue sacerdote formado en el Seminario de Barbastro, del que más tarde fue profesor, y posteriormente fue Conservador del Patrimonio Artístico de la diócesis de Barbastro-Monzón y director del Museo Diocesano de Barbastro. Era además natural de Bonansa, pueblo cercano a Obarra, y por tanto buen conocedor de la zona.

Sea como sea, la aparición de la — por otra parte espléndida- fotografía de Del Arco permite ahora por fin llevar a cabo una edición definitiva de la inscripción y un estudio fiable sobre su paleografía, así como asegurar su datación (Fig. 4).

20 DEL ARCO, Ricardo, Fotografías de historia y arte. 1914-1924. Diputación Provincial de Huesca, Huesca, 2009, p. 176

21 En el portal de Internet DARA-Documentos y Archivos de Aragón aparece la fotografía, con una fecha orientativa 1913-1924. Agradecemos a Joan Casimiro esta información, así como algunas referencias bibliográficas.

22 DEL ARCO, Ricardo, Algunos datos..., p. 444.

${ }_{23}$ PONS, Jordi, «Alguns aspectes de la Ribagorça de l'època romana a través de l'epigrafia i la documentació visigótica i medieval», Empúries, 45-46, 1983-1984, p. 229.

24 DEL ARCO, Ricardo, Catálogo Monumental de España. Huesca. Madrid, 1942, pp. 43-44.

25 IGLESIAS COSTA, Manuel, El Monasterio..., p. 4. 


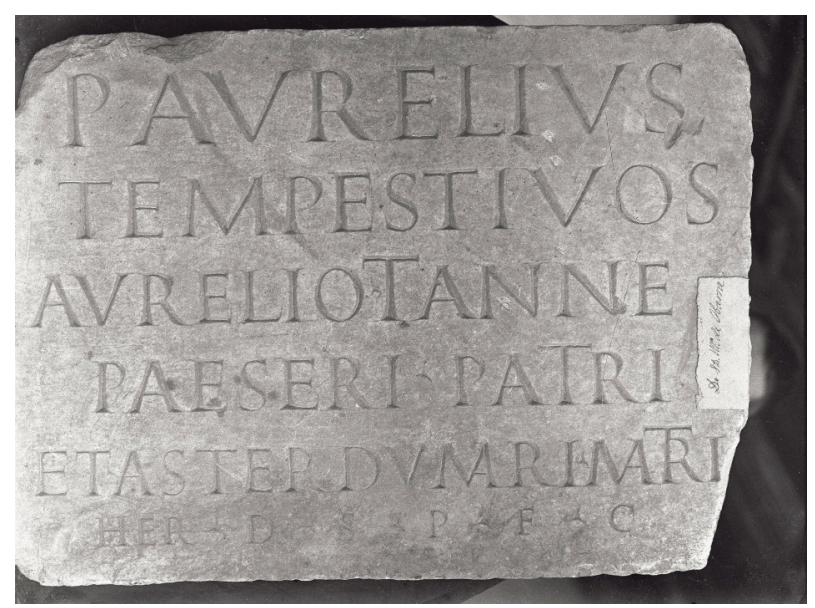

Fig. 3. COSTA, J.: op. cit. p. 90.

Así, el texto debería transcribirse definitivamente como sigue:

$P($ ublius $) \cdot$ Aurelius

Tempestivos

Aurelio - Tanne-

paeseri · patri

5 et $\cdot$ Asterdumari $\cdot \hat{m a t h i}$

her(es) $\cdot d(e) \cdot s($ ua $) \cdot p($ ecunia $) \cdot f($ aciendum $) \cdot c($ uravit)

Desde el punto de vista paleográfico hay que constatar la excelente ordinatio de los renglones, casi todos centrados con respecto al margen (obsérvese en especial I. 4), aunque probablemente el apuro de espacio es el que ha provocado el recurso a los nexos al final de la I. 5. Los signos han sido grabados con incisión homogénea y tienen regularmente remates. Como ya dejaba ver el dibujo de Costa, las T de las líneas 3 y 4 son de mayor módulo; la P no cierra el óculo. Las interpunciones no son exactamente triangulares, sino en forma de estrella de tres puntas dirigidas hacia arriba. Tanto las características paleográficas como la grafía Tempestivos invitan a fechar la inscripción en época augústea o, como muy tarde, julio-claudia, quizás sólo unos decenios antes de lo que el excelente criterio epigráfico de Hübner había atinado a proponer, como se ha visto antes, aun sin ver la pieza.

Por lo demás, la fotografía ratifica la corrección de la lectura de la pieza que se deriva de Costa en detrimento de la tradición que parte de Abad y Lasierra y recogen Fita y Hübner. Ello es especialmente importante para confirmar la forma del cognomen Tempestivos del dedicante — no muy abundante en el repertorio, dicho 
sea de paso-, pero también la de los cognomina de sus progenitores, Tannepaeseri y Asterdumari, que han merecido ya notable atención por parte de los estudiosos de la onomástica paleohispánica ${ }^{26}$. En resumidas cuentas, todos estos aspectos epigráficos, onomásticos y de otro tipo podrán ser en adelante abordados y discutidos desde la seguridad que proporciona la excelente fotografía ahora recuperada.

26 Véase al respecto UNTERMANN, J., Monumenta Linguarum Hispanicarum III.1, Wiesbaden, 1990, 232. 213. 211 
